\title{
Induction of colon tumorigenesis by glutathione depletion in p53-knock-out mice
}

\author{
JOHN P. RICHIE Jr ${ }^{1}$, DESPINA KOMNINOU ${ }^{2}$ and ANTHONY P. ALBINO ${ }^{3}$ \\ ${ }^{1}$ Department of Health Evaluation Sciences and Pharmacology, Penn State Cancer Institute, \\ Penn State University College of Medicine, Hershey, PA 17033; ${ }^{2}$ Former Institute for Cancer Prevention, \\ American Health Foundation Cancer Center, Valhalla, NY 10595, USA
}

Received December 11, 2006; Accepted January 15, 2007

\begin{abstract}
In order to examine the role of glutathione (GSH), a key cellular antioxidant, on spontaneous tumor development, we tested the effects of buthionine sulfoximine (BSO), a specific inhibitor of GSH synthesis, and 1,2-oxothiazolidine4-carboxylic acid (OTCA), a cysteine and GSH precursor, on tumor incidence and spectrum in p53 nullizygous (p53--) transgenic mice. Mice were randomly assigned to three groups: control (no treatment), BSO $(20 \mathrm{mM}$ in drinking water) or OTCA $(6 \mathrm{~g} / \mathrm{kg}$ in the diet) $(\mathrm{n}=30$ per group). After 10 weeks, GSH levels were decreased $29-88 \%$ in all tissues except liver and brain in BSO-treated mice, while no changes were observed in most tissues from OTCA-treated animals. Mice in all groups showed similar survival patterns as well as incidence of the most commonly observed tumors: i.e., lymphomas (80\%) and other tumors (38\%). However, a 5fold increase in incidence of colonic tumors (from 4-20\%) was observed in the BSO-treated group, suggesting that GSH deficiency and loss of p53 function play contributory roles in colon carcinogenesis.
\end{abstract}

\section{Introduction}

Glutathione ( $\gamma$-Glu-Cys-Gly) (GSH) is a ubiquitous intracellular thiol which functions as a major endogenous antioxidant and redox buffer $(1,2)$. GSH has numerous other

Correspondence to: Dr John P. Richie, Department of Health Evaluation Sciences and Pharmacology, Penn State Cancer Institute, Penn State University College of Medicine, Hershey, PA 17033, USA E-mail: jrichie@psu.edu

Present address: ${ }^{3}$ Vector Group Ltd., 712 Fifth Avenue, New York, NY 10019, USA

Abbreviations: BSO, buthionine sulfoximine; DTNB, 5,5' dithiobis(2-nitrobenzoic acid); GSH, glutathione; MPA, metaphosphoric acid; OTCA, 1,2-oxothiazolidine-4-carboxylic acid; PCNA, proliferating cellular nuclear antigen

Key words: glutathione, buthionine sulfoximine, p53, colon cancer well-established functions including the detoxification of carcinogens, xenobiotics and lipid peroxides, the regulation of protein function and gene expression via alteration of redox sensitive trans-acting factors (3-7).

Administration of GSH inhibited tumor formation in the murine skin multistage carcinogenesis model (8), in aflatoxin-induced rat hepatocellular carcinomas (9), and in DMBA-induced oral tumors in hamsters (10). Likewise, manipulations that increase cellular GSH stores such as the administration of the GSH precursors, $\mathrm{N}$-acetylcysteine or 1,2-oxothiazaladine-4-carboxylic acid (OTCA) have proven effective in inhibiting tumor formation at many sites $(11,12)$. Conversely, depletion of GSH stores have been associated with increases in cell susceptibility to oxidative damage (13) and potentiation of aflatoxin B1-induced liver tumors (14) and benzo[a]-pyrene-induced pulmonary and forestomach tumors (15) in animal models.

Based upon the many protective roles of GSH, we postulated that GSH depletion represents a key risk factor for human carcinogenesis (16). In laboratory animals and humans, glutathione depletion occurs as a result of heavy alcohol consumption $(17,18)$, poor nutrition (19), drug and xenobiotic toxicity $(20)$, and during aging $(16,21,22)$, factors which are often related to increased cancer susceptibility at many sites.

In the present study, we examined if specific alterations in GSH levels would result in changes in the onset of spontaneous tumor formation and/or alter the spectrum of induced tumors in the p53-deficient mouse model. The p53 deficient (knock-out) mouse has been utilized as an important model of tumorigenesis. The p53 tumor suppressor gene acts to prevent the propagation of DNA damage within the cell by instigating a diverse set of biological phenomena in response to genomic damage, including transient suppression of cell cycle progression, induction of apoptosis, activation of DNA repair genes, and modulation of DNA replication and transcription (23). Cells with inactivated, mutated, or missing p53 lack one or more of these functions, thereby promoting genetic instability, inappropriate cellular responses to DNAdamaging agents such as ROS and chemotherapeutic agents, and eventual tumor formation. Mice in which both alleles of the $p 53$ gene have been deleted are highly susceptible to a variety of spontaneous tumors at an early age and to premature death (24). GSH levels were depleted using buthionine sul- 
foximine (BSO), a specific inhibitor of GSH synthesis, and oxothiazolidine-carboxylate (OTCA), a cysteine precursor and promoter of GSH synthesis was used to enhance GSH status.

\section{Materials and methods}

Animals and diets. Ninety male TSG-p53 $3^{-/}$knock-out mice (4-5 weeks of age) were obtained from Taconic Labs (Germantown, NY). The derivation and tumor susceptibilities have been described (24). Mice were individually housed in polycarbonate cages on hardwood bedding and were maintained on a 12-h light/dark cycle at $24^{\circ} \mathrm{C}$ and fed an AIN-76A semi-purified diet ad libitum (AL). Mice were also allowed unlimited access to distilled water. After a 2week acclimatization period, animals were randomly assigned to 3 groups: control, BSO and OTCA. Group 1, serving as a control, were fed the AIN-76A diet AL and given water AL. Mice in group 2 received AIN-76A diet and water supplemented with $2.5 \mathrm{mM}$ BSO AL. After 5 weeks, the concentration of BSO in the drinking water was increased to $20 \mathrm{mM}$ in this group. Mice in group 3 received the AIN-76A diet supplemented with $6 \mathrm{~g} / \mathrm{kg}$ OTCA and water AL. Body weight was recorded weekly and water consumption was monitored periodically throughout the study.

Tumorigenesis studies and histopathological evaluation of tumors. All mice were observed daily for tumor formation by a qualified animal technologist (AALAS certified) throughout the test period. Animals that were moribund or were sacrificed as scheduled were necropsied under the supervision of a pathologist. The gross necropsy included an initial physical examination of the external surfaces and all orifices followed by an internal examination of tissues and organs in situ. The entire mucosal surfaces of the esophagus, stomach, small and large intestines, and rectum were opened and examined before fixation. All gross lesions were recorded in narrative, descriptive terms, including location, size number, shape, color, and texture. All tissues and organs were immediately preserved in $10 \%$ buffered formalin. Tissue fixation and trimming and slide preparation for histology were done as described in the NCI Carcinogen Bioassay Guidelines [DHEW Publication No. (NIH 76-801)]. Multiple sections were made on each tissue or organ in which a tumor was suspected. The pathologist recorded all microscopic findings, including all abnormalities, lesions, neoplasms, metastatic tumors and their anatomic location. Also, at the end of 10 weeks, 5 animals randomly selected from each group were sacrificed by $\mathrm{CO}_{2}$ between 8:00 and 9:00 am. Tissues were immediately removed and rinsed in ice-cold saline, trimmed and weighed. Tissue samples were homogenized for GSH analysis and the remainder stored at $-70^{\circ} \mathrm{C}$. Tissue homogenates $(10 \% \mathrm{w} / \mathrm{v})$ were prepared in cold $5 \%$ metaphosphoric acid (MPA). After centrifugation at 13,000 $\mathrm{x}$ g for $2 \mathrm{~min}$, the extracts were stored at $-70^{\circ} \mathrm{C}$ for less than 1 week until analysis.

Analysis of glutathione. Glutathione was measured in MPA extracted samples using our micro-plate method (25), based on the 5,5'dithiobis-(2-nitrobenzoic acid) (DTNB)/enzymatic recycling procedure of Tietze (26), and Owens and Belcher (27).

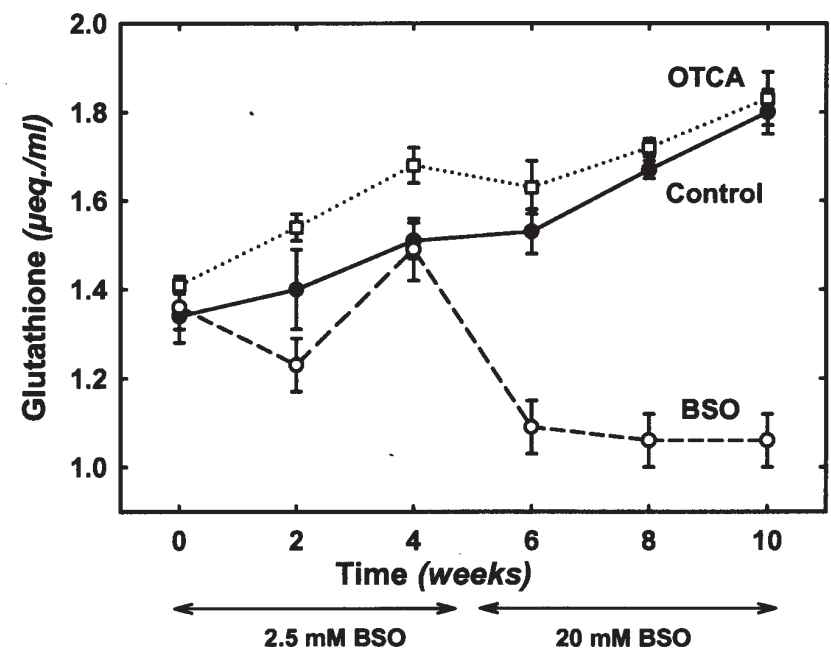

Figure 1. Blood glutathione depletion in BSO-treated p53 knock-out mice. Retro-orbital blood samples were obtained from mice in 3 groups: control, BSO (provided in drinking water) and OTCA (provided in diet) (5 mice per group). Samples were processed and GSH analyzed as described in text.

Statistical analysis. Body weights, tumor incidence, tumor multiplicity, and GSH levels were compared between animals using analysis of variance (28). Tumor incidence expressed as percentage of mice with tumors and was compared between the groups by Chi-squared analysis.

\section{Results}

Effects of BSO and OTCA on GSH levels in p53-deficient mice. In a subset consisting of 5 mice per group, blood GSH levels were monitored bi-weekly in retro-orbital blood samples over the first 10 weeks of the study (Fig. 1). A progressive 30 to $35 \%$ increase in GSH levels were observed in both control and OTCA-treated mice over the 10 -week period. Over the first 4 weeks, GSH levels tended to be higher in the OTCA group with a significant $15 \%$ difference observed at 4 weeks $(\mathrm{p}<0.05)$. However, no differences were observed between the 2 groups thereafter. Blood GSH levels of mice in the BSO group were the same as controls over the first 4 weeks when they were receiving $2.5 \mathrm{mM}$ BSO in their drinking water. At each time point thereafter, when the animals were receiving $20 \mathrm{mM}$ BSO, GSH levels were reduced to approximately $1.05 \mathrm{mM}, 30-40 \%$ lower that those observed in either control or OTCA-treated mice.

After 10 weeks all 5 animals per group were sacrificed and tissue GSH levels determined (Table I). No differences were observed between control and OTCA-treated mice for most of the tissues examined. In contrast, significantly lower levels were observed for all tissues except liver and brain in the BSO-treated animals. BSO-induced decreases in GSH ranged from $88 \%$ in the colon to $29 \%$ in the spleen. Liver levels were unchanged by BSO-treatment after 10 weeks.

Growth and survival of p53 deficient mice. Growth curves were similar for both control and OTCA treated mice throughout the course of the study (Fig. 2). However, BSO had a significant effect on body weight, beginning at 5 weeks when 
Table I. Tissue glutathione levels in p53 knock-out mice after 10 weeks of BSO or OTCA administration.

\begin{tabular}{lccc}
\hline & \multicolumn{3}{c}{ GSH + GSSG $(\mu \text { eq./g tissue or } \mathrm{ml} \text { blood })^{\mathrm{a}}$} \\
\cline { 2 - 4 } Tissue & Control & BSO $(\%)$ & OTCA $(\%)$ \\
\hline Whole blood & $1.80 \pm 0.05$ & $1.06 \pm 0.06^{\mathrm{b}}$ & $1.83 \pm 0.06$ \\
& & $(59)$ & $(102 \%)$ \\
Liver & $6.21 \pm 0.69$ & $5.87 \pm 0.11$ & $5.95 \pm 0.49$ \\
& & $(94)$ & $(96)$ \\
Kidney & $2.01 \pm 0.20$ & $0.57 \pm 0.14^{\mathrm{b}}$ & $2.33 \pm 0.11^{\mathrm{b}}$ \\
& & $(28)$ & $(116)$ \\
Heart & $0.85 \pm 0.16$ & $0.15 \pm 0.04^{\mathrm{b}}$ & $0.99 \pm 0.08$ \\
& & $(18)$ & $(116)$ \\
Colon & $1.80 \pm 0.16$ & $0.22 \pm 0.05^{\mathrm{b}}$ & $1.88 \pm 0.07$ \\
& & $(12)$ & $(104)$ \\
Oral mucosa & $1.11 \pm 0.23$ & $0.28 \pm 0.01^{\mathrm{b}}$ & $1.18 \pm 0.16$ \\
& & $(25)$ & $(106)$ \\
Spleen & $2.48 \pm 0.08$ & $1.77 \pm 0.09^{\mathrm{b}}$ & $2.95 \pm 0.06^{\mathrm{b}}$ \\
& & $(71)$ & $(119)$ \\
Brain & $1.43 \pm 0.16$ & $1.32 \pm 0.05$ & $1.28 \pm 0.16$ \\
& & $(92)$ & $(90)$ \\
\hline
\end{tabular}

${ }^{a}$ Values are mean \pm SEM $(\%$ of control $), n=5$. ${ }^{\text {SSignificantly different }}$ from control group $(\mathrm{P}<0.05)$.

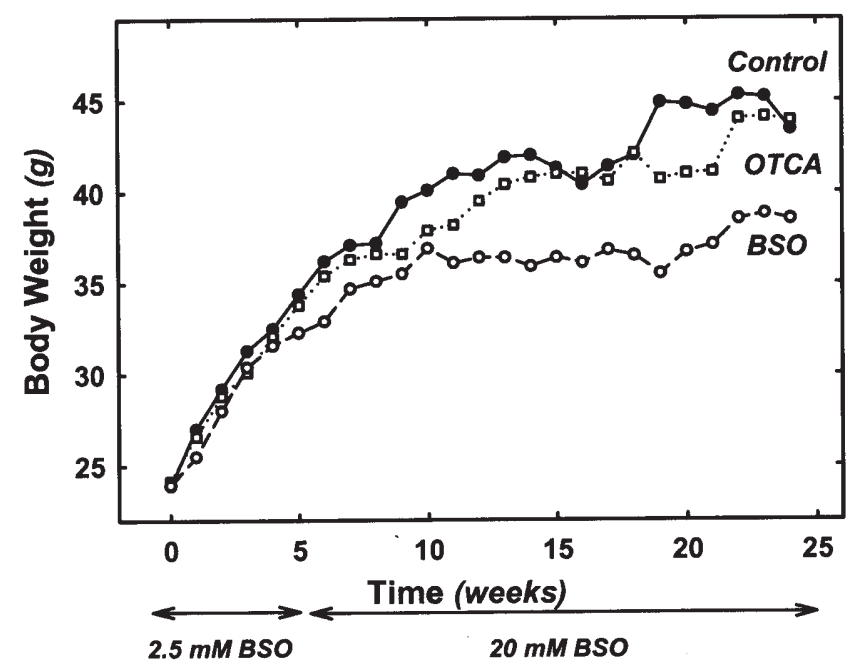

Figure 2. BSO and OTCA effects on body weight in p53-deficient mice.

the BSO concentration was increased from 2.5 to $20 \mathrm{mM}$. During the initial $2.5 \mathrm{mM}$ BSO period, body weight was identical between all 3 groups. Once the BSO concentration was increased to $20 \mathrm{mM}$, a decreased growth rate was observed compared to controls until 10 weeks of age where growth was virtually halted in the BSO animals. Thus, the

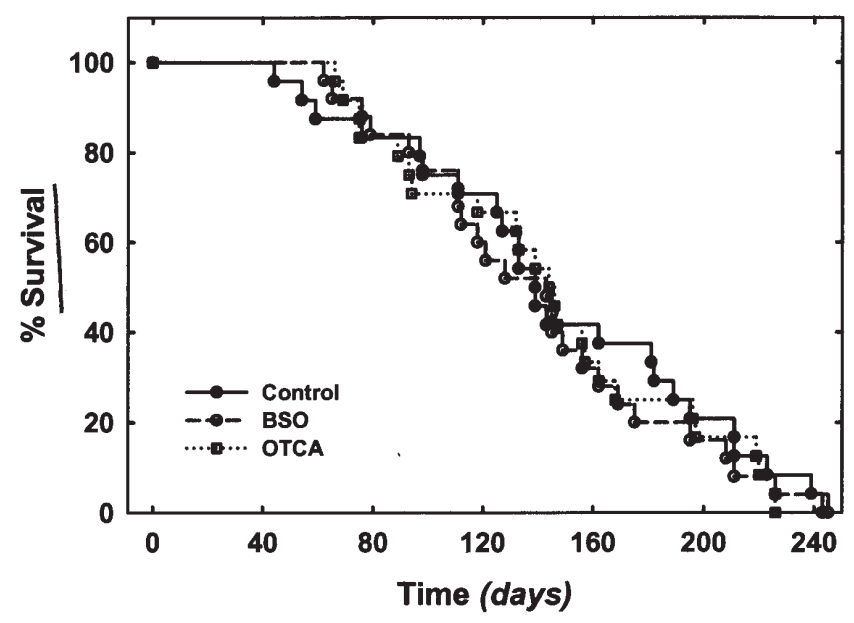

Figure 3. Survival of p53 knock-out mice.

Table II. Effect of BSO and OTCA on tumor development in p53-deficient mice.

\begin{tabular}{lccc}
\hline & Control & BSO & OTC \\
\cline { 2 - 4 } Lymphomas $^{\mathrm{a}}$ & & & \\
Thymus & 58.6 & 56.7 & 48.3 \\
Spleen & 37.9 & 46.7 & 34.5 \\
Total & 82.8 & 80.0 & 79.3 \\
Survival (days) & $148 \pm 56$ & $135 \pm 50$ & $145 \pm 52$ \\
Colon tumors $^{\mathrm{a}}$ & 4.0 & $20.0^{\mathrm{c}}$ & 8.0 \\
Survival (days) $^{\mathrm{b}}$ & 171 & $134 \pm 41$ & $163 \pm 54$ \\
Other tumors $^{\mathrm{a}}$ & 37.9 & 50.0 & 41.4 \\
Survival (days) $^{\mathrm{b}}$ & $170 \pm 40$ & $112 \pm 30$ & $126 \pm 55$ \\
\hline
\end{tabular}

a\% of mice. ${ }^{b}$ Values are mean \pm SD. ${ }^{c}$ Significantly different from control group, $\mathrm{P}<0.05$.

initial 5-week period with $2.5 \mathrm{mM}$ BSO was not effective at preventing the later loss in body weight. Over this later period, mean body weights were 15 to $20 \%$ lower in BSOtreated animals.

Survival curves for all three groups of p53-deficient mice (control, BSO-treated and OTCA-treated) are provided in Fig. 3. The earliest death occurred at 44 days and the latest at 245 days. After the initial 6 weeks of the study, death rates were linear in all groups. Most importantly, no differences were observed in survival between groups throughout the 34-week study period.

Effects of BSO and OTCA on spontaneous tumor development in p53-deficient mice. A summary of the time course and spectrum of spontaneous tumor development in mice from all groups is provided in Table II. The spectrum and time of occurrence of tumors previously observed in control animals were similar to those observed for p53 deficient mice (29). Tumors were found in all animals with about $40 \%$ of mice presenting with multiple tumor types. The most commonly 
observed tumors were lymphomas (80\%), and most of these involved the thymus $(60 \%)$ and/or the spleen $(45 \%)$. Other tumors, including sarcomas and hepatocarcinomas, were observed in $38 \%$ of control mice. No differences were observed in the occurrence or timing of any of the predicted tumor types between control mice in either BSO or OTCA-treated animals.

In contrast to these, premalignant and malignant colon tumors are relatively uncommon in this p53-deficient strain. Pathological examination revealed the presence of adenomatous polyps in $4 \%$ of control mice (Table II). While the occurrence of colonic tumors in OTCA-treated mice did not differ significantly from control animals, a 5-fold increase was observed in BSO-treated animals. Specifically, the incidence of adenomas and adenocarcinomas in the BSO-treated group was increased by 3 -fold (from 4-2\%) and 8-fold (from 0-8\%) respectively, compared to controls. The observed adenocarcinomas were well differentiated and invaded the muscularis mucosa while the adenomas were benign with moderate epithelial atypia. No differences in the time of occurrence of these tumors were detected between groups.

\section{Discussion}

GSH depletion has numerous effects on key cellular processes involved in the malignant conversion of cells including enhanced generation of ROS, induction of oxidative damage and decreased carcinogen detoxification capacity. High cellular levels of GSH, thus, may serve as a key protective mechanism against genetic damage by reactive genotoxic agents as well as affecting post-initiation phases of carcinogenesis. We sought to determine the direct effects of specifically modulating the tissue levels of GSH on tumor incidence and spectrum in the p53-deficient mouse which develops spontaneous tumors at several organ sites at an early age. Our results indicate that lowering the level of GSH by BSO administration does not accelerate tumor onset but does increase the incidence of colon carcinogenesis in these mice by 5 -fold. Conversely, administration of OTCA had little effect on steady state GSH levels and tumorigenesis.

Due to its specificity for reducing GSH levels, low toxicity and effectiveness when administered in drinking water, BSO, a specific inhibitor of $\gamma$-glutamylcysteine synthetase, the rate-limiting enzyme for GSH synthesis, is well suited for chronic studies of GSH depletion $(30,31)$. Our results demonstrate the effectiveness of BSO in the drinking water at depleting tissue GSH levels over long periods of time and in controlling tissue GSH at specific desired levels. It is of interest to note that after 10 weeks of administration, BSO had its greatest effect on colon tissue ( $88 \%$ depletion).

A $15-20 \%$ decrease in body weight was observed in p53deficient mice treated with $20 \mathrm{mM}$ BSO. However, there were no other observable indications of BSO-related toxicity in these mice. While the factors responsible for this reduction in body weight are unknown, we do not believe that this phenomenon affects the conclusions drawn from the studies. In a previous report, a decrease in body weight of nearly $50 \%$ by caloric restriction was associated with a significant decrease in the incidence of hematopoietic neoplasias and sarcomas and increased survival in p53-deficient mice (29).
The major effects of GSH deficiency induced by BSO from previous study include mitochondrial damage to liver, kidney, lung, heart and lens of mice, but only after mitochondrial GSH levels were depleted by greater than $50 \%$ (32). BSO induced structural damage to cells only when GSH levels were depleted by $>97 \%$ for 3 weeks in mice (33).

Consistent with previous reports $(24,29)$, the major tumor types observed in all groups of p53-deficient mice were hematopoietic neoplasms and sarcomas. As a result of the rapid progression and lethality associated with these tumors, the mean lifespan of these animals was limited to approximately 15 weeks. Unlike calorie restriction, which has been shown to delay the onset of these specific tumor types and consequently enhance survival slightly in p53-deficient mice (29), GSH depletion by BSO had no effect on either the onset of these tumors or overall survival. Thus the results presented here suggest that the loss of p53 is clearly the dominant factor in the etiology of these hematopoietic neoplasms and the absence of GSH plays, at best, only a minor role.

The damage induced by genotoxic agents and ROS are commonly thought to occur over long-periods of time and, thus, primarily impact on the induction and promotion of age-related tumors occurring later in life. Therefore, it is likely that the cellular protection afforded by GSH primarily impacts only those late occurring tumor types. The predominant tumor types occurring in the p53-deficient mouse are mainly lymphoid, which usually occur early in life in the human and are not associated with aging. It is perhaps not surprising, therefore, that GSH depletion has no substantial effect on the onset or lethality of these tumors. Moreover, because of the rapid tumor development and short lifespan of p53-deficient mice, few late developing, age-related tumors (such as colon cancer) are observed. Accordingly, colon tumors were observed in only $4 \%$ of control animals. Thus, our finding of 5-fold increase in incidence of colon tumors as a result of GSH depletion represents a significant enhancement in risk.

Colon cancer development and progression is believed to involve the systematic activation of oncogenes and inactivation of tumor suppressor genes, including p53 (34), that results in a series of phenotypic cellular states displaying progressive dysplasia and enhanced proliferation (35). Specific roles for oxidative damage and GSH depletion in this process are suggested by numerous reports demonstrating that antioxidants and other GSH-related compounds are effective inhibitors of colon carcinogenesis in relevant colon tumor models (36). In the rat, dietary supplementation with specific GSH enhancing agents $\mathrm{N}$-acetylcysteine or OTCA resulted in a reduction in the number of azoxymethane-induced aberrant crypt foci (ACF) in the colon (12). Finally, molecular epidemiology studies have suggested that individuals who possess the null phenotype for the GSH-related detoxification enzyme GSTM1 are at increased risk for cancer at a number of sites including colon (37).

In the present studies, OTCA was ineffective at increasing tissue GSH levels and no differences were observed between OTCA animals and control mice in any parameter measured. OTCA, which is converted intracellularly to Cys, the ratelimiting precursor for GSH synthesis, has been shown to enhance tissue GSH levels when administered either by injection or in the diet (OTCA has relatively low toxicity and 
has been used as a Cys pro-drug for the intracellular delivery of Cys since Cys itself is toxic (38). However, in all reports in which OTCA was shown to enhance tissue GSH, the GSH levels were initially depleted by either fasting or administration of a GSH depleting agent. To our knowledge, there have been no reports of OTCA enhancing tissue GSH in tissues with normal GSH levels. This together with our present results suggests that OTCA is not an effective means of enhancing GSH levels in long-term studies.

In summary, these results indicate that a depletion of GSH and loss of p53 function may play contributory roles in colon carcinogenesis, perhaps involving the accumulation of oxidative damage over time. However, further mechanistic studies are needed to assess any pro-tumorigenic effects of GSH depletion, uncoupled from body weight changes, in the development of a useful model for spontaneous colon tumorigenesis.

\section{Acknowledgements}

We gratefully acknowledge Joel Reindhart and Jeffrey Rigotti for their technical help, and Dr Chung-Xiou Wang for her assistance in pathologic determinations. This research was supported by USPHS Grants CA17613 and AG14102.

\section{References}

1. Meister A and Anderson ME: Glutathione. Annu Rev Biochem 52: 711-760, 1983.

2. Viäna J: Glutathione: Metabolism and Physiological Functions. CRC Press, Boca Raton, 1990.

3. Coles B and Ketterer B: The role of glutathione and glutathione transferases in chemical carcinogenesis. Crit Rev Biochem Mol Biol 25: 47-70, 1990

4. Ketterer B: Protective role of glutathione and glutathione transferases in mutagenesis and carcinogenesis. Mutat Res 202: 343-361, 1988 .

5. Lu SC: Regulation of hepatic glutathione synthesis: current concepts and controversies. FASEB J 13: 1169-1183, 1999.

6. Hall AG: Review: the role of glutathione in the regulation of apoptosis. Eur J Clin Invest 29: 238-245, 1999.

7. Sies H: Glutathione and its role in cellular functions. Free Radic Biol Med 27: 916-921, 1999.

8. Rotstein JB and Slaga TJ: Effect of exogenous glutathione on tumor progression in the murine skin multistage carcinogenesis model. Carcinogenesis 9: 1547-1551, 1988.

9. Novi AM: Regression of aflatoxin B1-induced hepatocellular carcinomas by reduced glutathione. Science 212: 1547-1551, 1981.

10. Trickler D, Shklar G and Schwartz J: Inhibition of oral carcinogenesis by glutathione. Nutr Cancer 20: 139-144, 1993.

11. Estensen RD, Levy M, Klopp SJ, Galbraith AR, Mandel JS, Blomquist JA and Wattenberg LW: N-acetylcysteine suppression of the proliferative index in the colon of patients with previous adenomatous colonic polyps. Cancer Lett 147: 109-114, 1999.

12. Pereira MA and Khoury MD: Prevention by chemopreventive agents of azoxymethane-induced foci of aberrant crypts in rat colon. Cancer Lett 61: 27-33, 1991.

13. Ellouk-Achard S, Levresse V, Martin C, Pham-Huy C, DutertreCatella H, Thevenin M, Warnet JM and Claude JR: Ex vivo and in vitro models in acetaminophen hepatotoxicity studies. Relationship between glutathione depletion, oxidative stress and disturbances in calcium homeostasis and energy metabolism. Arch Toxicol Suppl 17: 209-214, 1995.

14. Hiruma S, Kimura M, Lehmann K, Gopalan-Kriczky P, Qin GZ, Shinozuka H, Sato K and Lotlikar PD: Potentiation of aflatoxin B1-induced hepatocarcinogenesis in the rat by pretreatment with buthionine sulfoximine. Cancer Lett 113: 103-109, 1997.
15. Srivastava SK, Xia H, Pal A, Hu X, Guo J and Singh SV: Potentiation of benzo[a]pyrene-induced pulmonary and forestomach tumorigenesis in mice by D,L-buthionine-S, Rsulfoximine-mediated tissue glutathione depletion. Cancer Lett 153: 35-39, 2000

16. Richie JP Jr: The role of glutathione in aging and cancer. Exp Gerontol 27: 615-626, 1992 .

17. Videla L and Guerri C: Glutathione and alcohol. CRC Press, Boca Raton, 1990

18. Lauterburg BH and Velez ME: Glutathione deficiency in alcoholics: risk factor for paracetamol hepatotoxicity. Gut 29: 1153-1157, 1988

19. Bray TM and Taylor CG: Tissue glutathione, nutrition, and oxidative stress. Can J Physiol Pharmacol 71: 746-751, 1993.

20. Jollow DJ: Glutathione thresholds in reactive metabolite toxicity. Arch Toxicol Suppl 3: 95-110, 1980.

21. Lang CA, Naryshkin S, Schneider DL, Mills BJ and Lindeman RD: Low blood glutathione levels in healthy aging adults. J Lab Clin Med 120: 720-725, 1992.

22. Julius M, Lang CA, Gleiberman L, Harburg E, Di Franceisco W and Schork A: Glutathione and morbidity in a community-based sample of elderly. J Clin Epidemiol 47: 1021-1026, 1994.

23. Levine AJ: p53, the cellular gatekeeper for growth and division. Cell 88: 323-331, 1997.

24. Donehower LA, Harvey M, Slagle BL, McArthur MJ, Montgomery CA Jr, Butel JS and Bradley A: Mice deficient for p53 are developmentally normal but susceptible to spontaneous tumours. Nature 356: 215-221, 1992

25. Richie JP Jr, Skowronski L, Abraham P and Leutzinger Y: Blood glutathione concentrations in a large-scale human study. Clin Chem 42: 64-70, 1996.

26. Tietze F: Enzymic method for quantitative determination of nanogram amounts of total and oxidized glutathione: applications to mammalian blood and other tissues. Anal Biochem 27: 502-522, 1969.

27. Owens CW and Belcher RV: A colorimetric micro-method for the determination of glutathione. Biochem J 94: 705-711, 1965.

28. Snedecor GW and Cochran WG: Statistical methods. 7th edition Iowa State University Press, Ames, IW, 1980.

29. Hursting SD, Perkins SN, Brown CC, Haines DC and Phang JM Calorie restriction induces a p53-independent delay of spontaneous carcinogenesis in p53-deficient and wild-type mice. Cancer Res 57: 2843-2846, 1997.

30. Griffith OW and Meister A: Potent and specific inhibition of glutathione synthesis by buthionine sulfoximine (S-n-butyl homocysteine sulfoximine). J Biol Chem 254: 7558-7560, 1979.

31. Nath RG, Ocando JE, Richie JP Jr and Chung FL: Effect of glutathione depletion on exocyclic adduct levels in the liver DNA of F344 rats. Chem Res Toxicol 10: 1250-1253, 1997.

32. Meister A: Glutathione deficiency produced by inhibition of its synthesis, and its reversal; applications in research and therapy. Pharmacol Ther 51: 155-194, 1991.

33. Martensson J and Meister A: Mitochondrial damage in muscle occurs after marked depletion of glutathione and is prevented by giving glutathione monoester. Proc Natl Acad Sci USA 86: 471-475, 1989.

34. Baker SJ, Fearon ER, Nigro JM, Hamilton SR, Preisinger AC, Jessup JM, van Tuinen P, Ledbetter DH, Barker DF, Nakamura Y, White R and Vogelstein B: Chromosome 17 deletions and p53 gene mutations in colorectal carcinomas. Science 244: 217-221, 1989.

35. Fearon ER and Vogelstein B: A genetic model for colorectal tumorigenesis. Cell 61: 759-767, 1990.

36. Wattenberg LW: Inhibition of carcinogenesis by minor dietary constituents. Cancer Res 52: S2085-S2091, 1992.

37. Zhong S, Wyllie AH, Barnes D, Wolf CR and Spurr NK: Relationship between the GSTM1 genetic polymorphism and susceptibility to bladder, breast and colon cancer. Carcinogenesis 14: 1821-1824, 1993.

38. Anderson ME and Meister A: Intracellular delivery of cysteine. Methods Enzymol 143: 313-325, 1987. 